\title{
On LQG Joint Optimal Scheduling and Control under Communication Constraints
}

\author{
Adam Molin and Sandra Hirche
}

\begin{abstract}
In this paper, we consider a discrete-time stochastic system, where sensor measurements are sent over a network to the controller. The design objective is a non-classical multicriterion optimization problem for finite horizon, where the cost function consists of the linear quadratic cost reflecting the control performance and a communication cost penalizing information exchange between sensor and controller. It is shown that the joint optimization of scheduling and control can be separated into three subproblems: an optimal regulator problem, an estimation problem and an optimal scheduling problem. The obtained results are extended to TCP-like networks with random packet loss. In the proposed framework, we classify three classes of schedulers, a purely randomized, a deterministic and a state-dependent scheme, and compare their performance by a numerical example.
\end{abstract}

\section{INTRODUCTION}

The issue of studying optimal stochastic control problems, where acquiring sensor measurements at the controller is costly, has already drawn attention in the 60 s and 70 s, for example in [1] and [2]. At that time, the main motivation for such consideration emerged in aerospace applications, where telemetry data of space vehicles has to be sent to a controlling ground station over a band-limited link. Recently, due to the advent of networked control systems, stochastic control problems with limited information exchange undergo a reinvigoration. Networked control systems can be defined as spatially distributed control systems with sensors, actuators and controllers exchanging information over a common digital communication network. There is an abundance of promising application areas identified as networked control systems, such as unmanned aerial vehicles [3], vehicular control networks [4] and teleoperation systems [5].

In presence of limited communication bandwidth or limited battery capacity in wireless networks, a major objective of networked control systems is to maximize control performance, while keeping information exchange over the feedback link at a minimum. In order to meet the tradeoff between such concurring objectives, basically three formulations as stochastic control problems have been devised within the linear quadratic Gaussian (LQG) framework taking into account communication constraints [1], [2], [6]-[17]. The first approach proposed in [6] takes an information theoretic viewpoint. It is assumed that sensor and controller are connected over a bit-rate constraint channel and conditions under which the separation principle of stochastic control

A. Molin and S. Hirche are with the Institute of Automatic Control Engineering, Technische Universität München, D-80290 München, Germany; http://www.lsr.ei.tum.de, adam.molindtum.de, s.hirchedieee.org holds are given. On the premise of the separation principle, the major task is then to develop optimal encoder-decoder pairs. In the second approach there is only a limited number of observations sent to the controller over a finite horizon. Determining the optimal timings of the observations to be sent is the main challenge in such approach. The procedure of assigning such timings will be called scheduling. In [1], [2], [7] timings are determined beforehand. Establishing scheduling rules that take into account current observations have been considered in [8]-[10] for discrete-time systems and in [11], [12] for continuous-time systems. In [13], it was shown that separation of estimation and control holds for stochastic discrete-time systems with a limitation on the number of control commands.

Rather than posing hard constraints on the information exchange, the third approach that is also considered in this paper introduces an additional communication cost term in the cost function. This term can be viewed as a penalty that has to be paid, whenever a measurement is transmitted to the controller. The scheduler situated at the sensor decides whether to transmit a measurement to the controller upon the available information. Such formulation has been considered in [14]-[16], but only for discrete-time estimation problems. An offline determination of the timing sequence for the joint optimal control and scheduling problem has been discussed in [17], where it is argued that assigning the scheduling sequence beforehand, the remaining problem reduces to the standard LQG problem for time-varying systems. Hence, in this case, the problem can be divided into separate subproblems that can be solved sequentially.

The main contribution of this article is to solve the joint optimal scheduling and control problem by establishing a separation of the problem into tractable subproblems. The scheduler may depend on the current state of the system. The subproblems are identified as an optimal regulator problem, an estimation problem and an optimal scheduling problem, which can be solved by standard algorithms. It also turns out that the joint optimal estimation and scheduling problem in the proposed framework have an intrinsic coupling that has not been regarded before. Furthermore, we give an extension to TCP-like communication networks with packet dropouts as in [18], where successful transmissions of packets are acknowledged at the sensor. Three classes of schedulers are identified, that are a purely randomized, a deterministic and a state-dependent scheme. These types of schedulers are compared in a numerical example to demonstrate the performance gains of the optimal state-dependent solution with respect to the optimal solutions of the other two classes. 
The remainder of the paper is organized into five sections. Section II introduces the stochastic discrete-time system model and the design objective. In section III, the problem is reformulated into tractable subproblems. An extension of the results to networks with packet loss is given in section IV. In section $\mathrm{V}$ three different types of schedulers are introduced, which are compared in a numerical example in section VI.

Notation. In this paper, the operators $\operatorname{tr}[\cdot]$ and $(\cdot)^{\mathrm{T}}$ denote the trace and the transpose operator of a square matrix, respectively. The variable $P$ denotes the probability measure on the abstract sample space denoted by $\Omega$. The expectation operator is denoted by $E[\cdot]$ and the conditional expectation is denoted by $E[\cdot \mid \cdot]$.

\section{PROBLEM STATEMENT}

We consider the following stochastic time-invariant discrete-time system $\mathcal{P}$

$$
x_{k+1}=A x_{k}+B u_{k}+w_{k},
$$

where $A \in \mathbb{R}^{n \times n}, B \in \mathbb{R}^{n \times d}$. The variables, $x_{k}$ and $u_{k}$ denote the state and the control input and are taking values in $\mathbb{R}^{n}$ and $\mathbb{R}^{d}$, respectively, the system noise $w_{k}$ takes values in $\mathbb{R}^{n}$ and is an i.i.d. (independent identically distributed) zero-mean Gaussian distributed sequence with covariance matrix $C_{w}=\mathrm{E}\left[w_{k} w_{k}^{\mathrm{T}}\right]$. The initial state, $x_{0}$ is Gaussian with mean $\bar{x}_{0}$ and covariance $C_{x_{0}}=\mathrm{E}\left[\left(x_{0}-\bar{x}_{0}\right)\left(x_{0}-\bar{x}_{0}\right)^{\mathrm{T}}\right]$. Let $(\Omega, \mathcal{A}, \mathrm{P})$ denote the probability space generated by the initial state $x_{0}$ and noise sequence $\left(w_{k}\right)_{k}$.

System parameters and statistics are known to the scheduler and controller. It is assumed that the scheduler $\mathcal{S}$ situated at the sensor side can observe the complete state and decides, whether the controller $\mathcal{C}$ should be updated with the current state. The controller is assumed to be situated at the actuator side, which implies that only sensor and controller must exchange information over the network. The system model is illustrated in Fig. 1. The interconnection of sensor and controller can be stated as the following measurement equation, which differs from the standard LQG formulation:

$$
y_{k}=\delta_{k} x_{k}
$$

where $y_{k}$ is the available measurement at the controller and $\delta_{k} \in\{0,1\}$ is the scheduler output given by

$$
\delta_{k}= \begin{cases}1 & \text { measurement } x_{k} \text { sent } \\ 0 & \text { no measurement transmitted }\end{cases}
$$

Let the scheduler law $\mathrm{f}=\left(\mathrm{f}_{0}, \mathrm{f}_{1}, \ldots, \mathrm{f}_{T-1}\right)$ and the control law $\gamma=\left(\gamma_{0}, \gamma_{1}, \ldots \gamma_{T-1}\right)$ denote admissible policies for the finite horizon problem with horizon $T$ and

$$
\begin{aligned}
\delta_{k} & =\mathrm{f}_{k}\left(\mathcal{I}_{k}^{\mathcal{S}}\right), \\
u_{k} & =\gamma_{k}\left(\mathcal{I}_{k}^{\mathcal{C}}\right) .
\end{aligned}
$$

In order to guarantee that the problem is well-defined, we presume for convenience that $\mathrm{f}$ and $\gamma$ are measurable functions with respect to $\mathcal{A}$. The information patterns $\mathcal{I}_{k}^{\mathcal{S}}$ and $\mathcal{I}_{k}^{\mathcal{C}}$ of the scheduler and controller are given by their complete observable history, i.e.

$$
\begin{aligned}
\mathcal{I}_{k}^{\mathcal{S}}= & \left\{\bar{x}_{0}, x_{0}, \delta_{0}, y_{0}, u_{0}, x_{1}, \delta_{1}, y_{1}, u_{1}, \ldots,\right. \\
& \left.\delta_{k-1}, y_{k-1}, u_{k-1}, x_{k}\right\} \\
\mathcal{I}_{k}^{\mathcal{C}}= & \left\{\bar{x}_{0}, \delta_{0}, y_{0}, u_{0}, \delta_{1}, y_{1}, u_{1}, \ldots,\right. \\
& \left.\delta_{k-1}, y_{k-1}, u_{k-1}, \delta_{k}, y_{k}\right\} .
\end{aligned}
$$

For $k=0$, we have $\mathcal{I}_{k}^{\mathcal{S}}=\left\{\bar{x}_{0}, x_{0}\right\}$ and $\mathcal{I}_{k}^{\mathcal{C}}=\left\{\bar{x}_{0}, \delta_{0}, y_{0}\right\}$. With abuse of notation, we use $\mathcal{I}_{k}^{\mathcal{S}}$ and $\mathcal{I}_{k}^{\mathcal{C}}$ as sets, when referring to information patterns, and as vectors, when referring to the information state. As we assume that the control law $\gamma_{k}$ is deterministic and the behavior of the communication network $\mathcal{N}$ is also known to the scheduler, past transmitted sensor measurements and control inputs are known to the scheduler. In fact, it can be noticed that the information patterns at scheduler and controller are nested after having determined the next scheduling variable $\delta_{k}$, i.e. $\mathcal{I}_{k}^{\mathcal{C}} \subset\left\{\mathcal{I}_{k}^{\mathcal{S}}, \delta_{k}, y_{k}\right\}$. Roughly speaking, this is an indicator that, although we have a distributed structure, decisions are virtually made by a centralized instance maintaining the possibility that the separation principle of optimal control still holds contrary to the counterexample given in [19] for a distributed information pattern.

The design objective is to find admissible policies $\mathrm{f}$ and $\gamma$ that minimize the multi-objective criterion

$$
J(\mathrm{f}, \gamma)=\mathrm{E}\left[x_{T}^{\mathrm{T}} Q_{T} x_{T}+\sum_{k=0}^{T-1} x_{k}^{\mathrm{T}} Q x_{k}+u_{k}^{\mathrm{T}} R u_{k}+\lambda \delta_{k}\right]
$$

The weighting matrices $Q, Q_{T}$ are positive definite and $R$ is positive semi-definite. The positive factor $\lambda$ can be regarded as the weight of penalizing information exchange between sensor and controller.

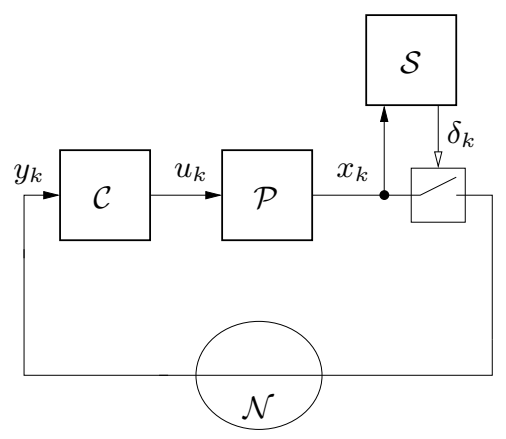

Fig. 1. System model of the networked control system with plant $\mathcal{P}$, scheduler $\mathcal{S}$, controller $\mathcal{C}$ and communication network $\mathcal{N}$.

\section{REFORMULATION}

Solving the optimization problem posed by the minimization of $J(f, \gamma)$ in (3) directly is a daunting task, since it involves joint optimization over real-valued functions $\gamma_{k}$ and discrete functions $\mathrm{f}_{k}$, whose number of input arguments increases with time $k$. In addition, due to the distributed information structure of controller and scheduler, the underlying problem with imperfect state information can not be 
transformed into a problem with perfect state information in a straightforward way as given in [20].

The aim of this section is to gradually transform the problem into equivalent problems that are more tractable without losing optimality.

For that reason, we first introduce the following equivalence classes of admissible pairs of scheduling and control policies. The equivalence class denoted by $[(\mathrm{f}, \gamma)]$ is defined in such way that any pair $(\hat{\mathrm{f}}, \hat{\gamma})$ within $[(\mathrm{f}, \gamma)]$ yields the same scheduling sequence $\left(\delta_{k}\right)_{k}$ as for the pair $(\mathrm{f}, \gamma)$ for any sample path $\omega \in \Omega$. Among all policy pairs within an equivalence class we will show that we can not get better than with a linear control policy obtained from the standard LQG problem. $[(\mathrm{f}, \gamma)]$ can be constructed by the map $\mathcal{T}$. Given a pair $(\mathrm{f}, \gamma)$ the equivalence class $[(\mathrm{f}, \gamma)]$ can be parameterized by the admissible control policies, which gives the corresponding scheduling policy

$$
\hat{\mathrm{f}}=\mathcal{T}(\mathrm{f}, \gamma, \hat{\gamma})
$$

where $\hat{\mathrm{f}}$ is adapted to control policy $\hat{\gamma}$ appropriately to satisfy the condition of having the same scheduling sequence for any sample path. It can be showed that the map $\mathcal{T}$ exists for any scheduling policy $\mathrm{f}$ and any admissible control policy $\gamma$ and $\hat{\gamma}$ as they are deterministic, measurable w.r.t. $\mathcal{A}$ and known to the respective scheduler by assumption. For a fixed control policy, it can be seen that the scheduling variable $\delta_{k}$ can be calculated by the information pattern $\hat{\mathcal{I}}_{k}^{S}=\left\{\bar{x}_{0}, x_{0}, w_{0}, w_{1}, \ldots, w_{k-1}\right\}$ at time $k$. With abuse of notation the scheduling policy $\hat{f}_{k}$ will be a function of $\hat{\mathcal{I}}_{k}^{S}$ in the following.

Subsequently, let $x_{k}^{\text {aug }}$ be the augmented state vector consisting of the plant state $x_{k}$ and the internal states $\hat{\mathcal{I}}_{k}^{\mathcal{S}}$ of the scheduler, let $Y^{k}=\left\{\bar{x}_{0}, y_{0}, \ldots, y_{k}\right\}, U^{k}=\left\{u_{0}, \ldots, u_{k}\right\}$, $\delta^{k}=\left\{\delta_{0}, \ldots, \delta_{k}\right\}$ be the observation history of the system with control policy $\hat{\gamma}$ and let $\tilde{Y}^{k}=\left\{\tilde{y}_{0}, \ldots, \tilde{y}_{k}\right\}$, $\tilde{\delta}^{k}=\left\{\tilde{\delta}_{0}, \ldots, \tilde{\delta}_{k}\right\}$ be the observation history assuming the control input to be the zero sequence.

Lemma 1: Given a system by (1), (2) and the scheduling policy $\hat{f}$ constructed by $\mathcal{T}$ from a fixed pair $(\mathrm{f}, \gamma)$ adapted to a used control policy $\hat{\gamma}$, the augmented state estimation error

$$
\Delta_{k}^{\text {aug }}=x_{k}^{\text {aug }}-\mathrm{E}\left[x_{k}^{\text {aug }} \mid Y^{k}, \delta^{k}, U^{k-1}\right]
$$

is independent of the used control policy $\hat{\gamma}$.

Proof: Fix a control policy $\hat{\gamma}$ and rewrite the system in the following augmented form with dynamics

$$
\left[\begin{array}{c}
x_{k+1} \\
\hat{\mathcal{I}}_{k+1}^{\mathcal{S}}
\end{array}\right]=\left[\begin{array}{c}
A x_{k}+B u_{k}+w_{k} \\
\left\{\hat{\mathcal{I}}_{k}^{\mathcal{S}}, w_{k}\right\}
\end{array}\right]
$$

and measurement equation

$$
y_{k}=H\left(\hat{\mathcal{I}}_{k}^{\mathcal{S}}, \delta_{k}\right)
$$

where $\hat{\mathcal{I}}_{0}^{\mathcal{S}}=\left\{\bar{x}_{0}, x_{0}\right\}$ and $\delta_{k}=\hat{\mathrm{f}}_{k}\left(\hat{\mathcal{I}}_{k}^{\mathcal{S}}\right)$. Similarly to [21], define the forced system

$$
\left[\begin{array}{c}
\bar{x}_{k+1} \\
0_{k+1}
\end{array}\right]=\left[\begin{array}{c}
A \bar{x}_{k}+B u_{k} \\
0_{k}
\end{array}\right]
$$

and the autonomous system

$$
\left[\begin{array}{c}
\tilde{x}_{k+1} \\
\hat{\mathcal{I}}_{k+1}^{\mathcal{S}}
\end{array}\right]=\left[\begin{array}{c}
A \tilde{x}_{k}+w_{k} \\
\left\{\hat{\mathcal{I}}_{k}^{\mathcal{S}}, w_{k}\right\}
\end{array}\right]
$$

with initial condition $\bar{x}_{0}$ for the forced and $x_{0}-\bar{x}_{0}$ for the autonomous part, respectively. The dummy variable $0_{k}$ has the same dimension of $\hat{\mathcal{I}}_{k}^{\mathcal{S}}$ containing only zero entries. Note that addition of (5) and (6) yields (4). Then, the error

$$
\begin{aligned}
\Delta_{k}^{\mathrm{aug}} & =\left[x_{k}^{\mathrm{T}}, \hat{\mathcal{I}}_{k}^{\mathcal{S}, \mathrm{T}}\right]^{\mathrm{T}}-\mathrm{E}\left[\left[x_{k}^{\mathrm{T}}, \hat{\mathcal{I}}_{k}^{\mathcal{S}, \mathrm{T}}\right]^{\mathrm{T}} \mid Y^{k}, \delta^{k}, U^{k-1}\right] \\
& =\left[\tilde{x}_{k}^{\mathrm{T}}, \hat{\mathcal{I}}_{k}^{\mathcal{S}, \mathrm{T}}\right]^{\mathrm{T}}-\mathrm{E}\left[\left[\tilde{x}_{k}^{\mathrm{T}}, \hat{\mathcal{I}}_{k}^{\mathcal{S}, \mathrm{T}}\right]^{\mathrm{T}} \mid Y^{k}, \delta^{k}, U^{k-1}\right] \\
& =\left[\tilde{x}_{k}^{\mathrm{T}}, \hat{\mathcal{I}}_{k}^{\mathcal{S}, \mathrm{T}}\right]^{\mathrm{T}}-\mathrm{E}\left[\left[\tilde{x}_{k}^{\mathrm{T}}, \hat{\mathcal{I}}_{k}^{\mathcal{S}, \mathrm{T}}\right]^{\mathrm{T}} \mid \tilde{Y}^{k}, \tilde{\delta}^{k}\right] .
\end{aligned}
$$

The second equality is due to the fact that $\bar{x}_{k}$ is measurable w.r.t. $U^{k-1}$. The third equality is because $\left[\tilde{x}_{k}^{\mathrm{T}}, \hat{\mathcal{I}}_{k}^{\mathcal{S}, \mathrm{T}}\right]^{\mathrm{T}}$ is independent of $U^{k-1}$. We observe from that expression that the error $\Delta_{k}^{\text {aug }}$ does not depend on $U^{k-1}$.

Remark 1: The dimension of the augmented state space is finite, since we consider problems with finite horizon. It should be noticed that we could have written Equation (4) in state space representation with a common state vector of constant dimensionality.

Remark 2: The above lemma is in accordance with [21], where it was shown that if a not completely controllable system has nonlinear observations in the uncontrollable subspace, the control may still have no dual effect. The condition of no dual effect states that the error covariance is independent of the control applied. The term dual comes from the dual role of the controller, i.e. (i) affecting state evolution and (ii) probing the system to reduce estimation uncertainty. The control is said to have no dual effect, if the latter probing property will have no effect.

It should be noted that Lemma 1 resembles Lemma 5.2.1 in section 5.2 of [20]. With the obtained results, a statement about the structure of the optimal controller can be given as follows:

Lemma 2: Given the system defined by (1), (2) and scheduler $\hat{f}$ constructed by $\mathcal{T}$ from any pair $(\mathrm{f}, \gamma)$ and adapted to a control policy yet to determine, the optimal control law $\gamma^{*}=\left(\gamma_{1}^{*}, \ldots, \gamma_{T-1}^{*}\right)$ minimizing $J(\mathcal{T}(\mathrm{f}, \gamma, \cdot), \cdot)$ is given by

$$
u_{k}=\gamma_{k}^{*}\left(\mathcal{I}_{k}^{\mathcal{C}}\right)=-L_{k} \mathrm{E}\left[x_{k} \mid \mathcal{I}_{k}^{\mathcal{C}}\right]
$$

with

$$
\begin{aligned}
L_{k}= & \left(R+B^{\mathrm{T}} P_{k+1} B\right)^{-1} B^{\mathrm{T}} P_{k+1} A, \\
P_{k}= & A^{\mathrm{T}} P_{k+1} A+Q-A^{\mathrm{T}} P_{k+1} B \\
& \times\left(R+B^{\mathrm{T}} P_{k+1} B\right)^{-1} B^{\mathrm{T}} P_{k+1} A, \\
P_{T}= & Q_{T},
\end{aligned}
$$

where $P_{k} \in \mathbb{R}^{n \times n}$ is non-negative definite for all $k$.

Proof: We notice that the communication cost is constant for any control policy by definition of $\hat{f}$. Thus, the cost $J(\mathcal{T}(\mathrm{f}, \gamma, \cdot), \cdot)$ in (3) reduces to the standard linear quadratic cost. Then, the derivation of the optimal control law $\gamma^{*}$ follows likewise as in section 5.2 in [20] by Lemma 1. 
Lemma 2 already gives a full characterization of the optimal control law. It states that if we have given an arbitrary admissible pair (f, $\gamma$ ), we can construct another pair $\left(\mathcal{T}\left(\mathrm{f}, \gamma, \gamma^{*}\right), \gamma^{*}\right)$ with $J\left(\mathcal{T}\left(\mathrm{f}, \gamma, \gamma^{*}\right), \gamma^{*}\right)$ less or equal to $J(\mathrm{f}, \gamma)$. This is due to the fact that the communication cost $\mathrm{E}\left[\sum_{k=0}^{T-1} \lambda \delta_{k}\right]$ are the same and the quadratic term is minimized by $\gamma^{*}$ due to Lemma 2 .

The remaining issue is how to calculate the conditional mean $E\left[x_{k} \mid \mathcal{I}_{k}^{\mathcal{C}}\right]$. The calculation can not be performed in a straight-forward way, as it is still coupled to the scheduling law due to $\mathcal{I}_{k}^{\mathcal{C}}$ which includes scheduling variables $\delta_{l}, l=0, \ldots, k$. On the assumption of having the optimal control law given by (7), we revise the cost function defined in (3). We will use the following identity that has been shown in [22] for the linear quadratic cost:

$$
\begin{aligned}
& J(\mathrm{f}, \gamma)=\mathrm{E}\left[x_{0}^{\mathrm{T}} P_{0} x_{0}+\sum_{k=0}^{T-1} w_{k}^{\mathrm{T}} P_{k+1} w_{k}\right. \\
& \left.+\sum_{k=0}^{T-1}\left(u_{k}+L_{k} x_{k}\right)^{\mathrm{T}}\left(R+B^{\mathrm{T}} P_{k+1} B\right)\left(u_{k}+L_{k} x_{k}\right)+\lambda \delta_{k}\right],
\end{aligned}
$$

where $L_{k}$ and $P_{k}$ are given by Lemma 2. In the following, let $J(\mathrm{f}, \gamma)=J^{\mathrm{C}}+J^{\mathrm{S}}(\mathrm{f}, \gamma)$, where

$$
\begin{aligned}
J^{\mathrm{C}}= & \mathrm{E}\left[x_{0}^{\mathrm{T}} P_{0} x_{0}+\sum_{k=0}^{T-1} w_{k}^{\mathrm{T}} P_{k+1} w_{k}\right], \\
J^{\mathrm{S}}(\mathrm{f}, \gamma)= & \mathrm{E}\left[\sum_{k=0}^{T-1}\left(u_{k}+L_{k} x_{k}\right)^{\mathrm{T}}\left(R+B^{\mathrm{T}} P_{k+1} B\right)\right. \\
& \left.\times\left(u_{k}+L_{k} x_{k}\right)+\lambda \delta_{k}\right] .
\end{aligned}
$$

We observe that $J^{\mathrm{C}}$ is constant, which implies it can be omitted in the optimization procedure. Due to this fact, we obtain the following optimization problem for the scheduling law, when taking the control law $\gamma^{*}$ given by (7)

$$
\begin{aligned}
\min _{f} \mathrm{E}[ & {\left[\sum_{k=0}^{T-1}\left(x_{k}-\mathrm{E}\left[x_{k} \mid \mathcal{I}_{k}^{\mathcal{C}}\right]\right)^{\mathrm{T}} L_{k}^{\mathrm{T}}\left(R+B^{\mathrm{T}} P_{k+1} B\right) L_{k}\right.} \\
& \left.\times\left(x_{k}-\mathrm{E}\left[x_{k} \mid \mathcal{I}_{k}^{\mathcal{C}}\right]\right)+\lambda \delta_{k}\right] .
\end{aligned}
$$

If the estimation error at the controller $\Delta_{k}=x_{k}-\mathrm{E}\left[x_{k} \mid \mathcal{I}_{k}^{\mathcal{C}}\right]$ forms a Markov chain controlled by $\delta_{k}$, then the problem can be posed in the dynamic programming framework with $\Delta_{k}$ as a sufficient statistic. Due to the information pattern $\mathcal{I}_{k}^{\mathcal{C}}$ appearing in $\Delta_{k}$, it can not be asserted a priori that $\Delta_{k}$ has the Markov property. Resolving this issue is the aim of the subsequent paragraph.

Our aim is to reduce the dimensionality of $\mathcal{I}_{k}^{\mathcal{C}}$, which appears in the conditional mean in (7). First, we observe that, for $\delta_{k}=1$, both controller and scheduler have common knowledge about the actual state $x_{k}$ at time $k$. Therefore, due to (2), we have $\mathrm{E}\left[x_{k} \mid \mathcal{I}_{k}^{\mathcal{C}}\right]=y_{k}=x_{k}$ for $\delta_{k}=1$. Let $\tau_{k}$ denote the last time instance where the state has been transmitted, when the current time instance is $k$, i.e. $\tau_{k}=\max \left\{l \mid \delta_{l}=1, l \leq k\right\}$. In case, no state has been transmitted before, we define $\tau_{k}=-1$. For convenience, we omit the index of $\tau_{k}$ in the following. The state at time $k$ with $\tau \geq 0$ can be written as

$$
x_{k}=A^{k-\tau} x_{\tau}+\sum_{l=\tau}^{k-1} A^{k-l-1}\left(B u_{l}+w_{l}\right) .
$$

In case $\tau=-1$, let $x_{-1}=\bar{x}_{0}, u_{-1}=0, w_{-1}=x_{0}-\bar{x}_{0}$ is defined as the uncertainty of the initial state. Thus, we have for $\tau=-1$

$$
x_{k}=A^{k}\left(x_{\tau}+B u_{\tau}+w_{\tau}\right)+\sum_{l=0}^{k-1} A^{k-l-1}\left(B u_{l}+w_{l}\right) .
$$

Consider the case where $\tau \geq 0$ and $k=\tau+1$

$$
\begin{aligned}
\mathrm{E}\left[x_{k} \mid \mathcal{I}_{k}^{\mathcal{C}}\right] & =\mathrm{E}\left[A x_{\tau}+B u_{\tau}+w_{\tau} \mid \mathcal{I}_{k}^{\mathcal{C}}\right] \\
& =\left(A-B L_{\tau}\right) x_{\tau}+\mathrm{E}\left[w_{\tau} \mid \mathcal{I}_{k}^{\mathcal{C}}\right] \\
& =\left(A-B L_{\tau}\right) x_{\tau}+\mathrm{E}\left[w_{\tau} \mid \delta_{\tau+1}=0\right] .
\end{aligned}
$$

The first equality is due to $x_{\tau}=y_{\tau} \in \mathcal{I}_{k}^{\mathcal{C}}$. The second is due to the fact that $w_{\tau}$ is independent to information gathered prior or at time $\tau$. It can easily be seen that the conditional mean for general $\tau \geq 0$ can be expressed as follows:

$$
\begin{aligned}
& \Delta_{\tau+l}=x_{\tau+l}-\mathrm{E}\left[x_{\tau+l} \mid \mathcal{I}_{\tau+l}^{\mathcal{C}}\right] \\
& =x_{\tau+l}-\mathrm{E}\left[x_{\tau+l} \mid x_{\tau}, \delta_{\tau+1}=0, \ldots, \delta_{\tau+l}=0\right] \\
& =\sum_{j=\tau}^{\tau+l-1} A^{\tau+l-j-1}\left(w_{j}-\mathrm{E}\left[w_{j} \mid \delta_{j+1}=0, \ldots, \delta_{\tau+l}=0\right]\right)
\end{aligned}
$$

For $\tau=-1$ similar results can be found.

The main observation is that the estimation of $x_{k}$ and the scheduling law are coupled through the terms $\mathrm{E}\left[w_{j} \mid \delta_{j+1}=\right.$ $\left.0, \ldots, \delta_{\tau+j}=0\right]$ in Equation (10), i.e. the choice of the scheduling law will influence the structure of the estimator. Fortunately, we will observe in the proof of Theorem 1 that these terms will vanish due to symmetry properties of the system.

The following theorem is the main result of this paper:

Theorem 1: The joint optimal controller and scheduling pair (f, $\gamma$ ) for minimizing the cost function $J$ defined in (3) is determined by the control law in (7) with estimator

$$
\mathrm{E}\left[x_{k} \mid \mathcal{I}_{k}^{\mathcal{C}}\right]= \begin{cases}x_{k} & \text { for } \delta_{k}=1 \\ \left(A-B L_{k}\right) \mathrm{E}\left[x_{k-1} \mid \mathcal{I}_{k-1}^{\mathcal{C}}\right] & \text { for } \delta_{k}=0\end{cases}
$$

where $\mathrm{E}\left[x_{0} \mid \mathcal{I}_{k}^{\mathcal{C}}\right]=\bar{x}_{0}$ for $\delta_{0}=0$ and by the solution of the optimization problem

$$
\begin{array}{r}
\min _{f} \mathrm{E}\left[\sum_{k=0}^{T-1}\left(1-\delta_{k}\right) e_{k}^{\mathrm{T}} \Gamma_{k} e_{k}+\lambda \delta_{k}\right], \\
\text { s.t. } e_{k+1}=\left(1-\delta_{k}\right) A e_{k}+w_{k},
\end{array}
$$

where $\Gamma_{k}=L_{k}^{\mathrm{T}}\left(R+B^{\mathrm{T}} P_{k+1} B\right) L_{k}$ and $e_{0}=x_{0}-\bar{x}_{0}$.

Proof: The structure of the optimal control law is already given by (7) due to Lemma 2 . In the remainder of the proof, we aim at showing that the sequence $\left(\Delta_{k}\right)_{k}$ is a Markov chain enabling us to transform the optimization problem in (9) to the problem given by (12). For that reason, 
we will show that the terms $\mathrm{E}\left[w_{j} \mid \delta_{j+1}=0, \ldots, \delta_{\tau+j}=0\right]$ will vanish in Equation (10).

First, consider the case, where $\tau=T-2$. Then, the remaining step $T-1$ is to solve the minimization

$$
\min _{\delta_{T-1} \in\{0,1\}} \mathrm{E}\left[\Delta_{T-1}^{\mathrm{T}} \Gamma_{T-1} \Delta_{T-1}+\lambda \delta_{T-1},\right]
$$

where $\Gamma_{k}=L_{k}^{\mathrm{T}}\left(R+B^{\mathrm{T}} P_{k+1} B\right) L_{k}$, for all $k=0, \ldots T-1$. As optimization (13) is equivalent to $\min \left\{\Delta_{T-1}^{\mathrm{T}} \Gamma_{T-1} \Delta_{T-1}, \lambda\right\}$ due to $\Delta_{T-1}=0$ for $\delta_{T-1}=1$, we have the following optimal scheduling rule

$$
\delta_{T-1}= \begin{cases}1 & \Delta_{T-1}^{\mathrm{T}} \Gamma_{T-1} \Delta_{T-1}>\lambda \\ 0 & \Delta_{T-1}^{\mathrm{T}} \Gamma_{T-1} \Delta_{T-1} \leq \lambda .\end{cases}
$$

Note that the scheduling law is not unique. It can differ arbitrarily on the boundary set $\Delta_{T-1}^{\mathrm{T}} \Gamma_{T-1} \Delta_{T-1}=\lambda$, since this set has Lebesgue measure 0 as $\Gamma_{k}$ is positive semidefinite and $\lambda$ is a positive real number. In order to cater for uniqueness, we define $\delta_{k}$ to be 0 on the boundary.

From (10), we have $\Delta_{T-1}=w_{T-2}-\mathrm{E}\left[w_{T-2} \mid \delta_{T-1}=0\right]$. Let $S_{T-1}$ be the set defined by

$$
\begin{aligned}
\left\{w_{T-2} \mid\left(w_{T-2}-\right.\right. & \left.\mathrm{E}\left[w_{T-2} \mid \delta_{T-1}=0\right]\right)^{\mathrm{T}} \Gamma_{T-1} \\
& \left.\times\left(w_{T-2}-\mathrm{E}\left[w_{T-2} \mid \delta_{T-1}=0\right]\right) \leq \lambda\right\} .
\end{aligned}
$$

The set $S_{T-1}$ basically defines the scheduling law for $T-1$. It can be observed that there virtually exists a degree of freedom in the choice for $\mathrm{E}\left[w_{T-2} \mid \delta_{T-1}=0\right]$. From a geometrical point of view, $\mathrm{E}\left[w_{T-2} \mid \delta_{T-1}=0\right]$ defines the position of the ellipsoid $S_{T-1}$ in $\mathbb{R}^{n}$, where $\mathrm{E}\left[w_{T-2} \mid \delta_{T-1}=0\right]$ is situated at the center, see (14). On the other hand, the choice of $S_{T-1}$ determines the value of E $\left[w_{T-2} \mid \delta_{T-1}=0\right]$, which may not be situated at the center. This recursive interdependence can only be resolved for $\mathrm{E}\left[w_{T-2} \mid \delta_{T-1}=0\right]=0$ due to symmetry properties of the Gaussian distribution. For all other choices of $\mathrm{E}\left[w_{T-2} \mid \delta_{T-1}=0\right]$, the center of $S_{T-1}$ and $\mathrm{E}\left[w_{T-2} \mid \delta_{T-1}=0\right]=0$ do not coincide, because of monotonicity properties of the Gaussian density function. By induction, it can be proved that $\mathrm{E}\left[w_{j} \mid \delta_{j+1}=0, \ldots, \delta_{\tau+j}=\right.$ $0]$ is zero for all $\tau$ and all $j$. Therefore, $\Delta_{k}$ is given by the recursive formulae

$$
\Delta_{k}= \begin{cases}0 & \text { for } \delta_{k}=1 \\ A \Delta_{k-1}+w_{k-1} & \text { for } \delta_{k}=0\end{cases}
$$

with $\Delta_{-1}=0$. Hence, the estimator is given by (11). In addition, it can be seen that Equations (9) and (15) are equivalent formulations to (12) which completes the proof.

Remark 3: We observe that the initial problem has been transformed into three subproblems which can be solved by standard numerical methods. Particularly, optimization problem (12) for determining the scheduling law can be solved by the dynamic programming algorithm [20].

Remark 4: The optimization given by (12) has been discussed in [15] as an average cost estimation problem with time-invariant weighting matrix $\Gamma_{k}$. In [16] an sub-optimal low-complexity algorithm has been derived for determining $f$ in the average cost sense to overcome the curse of dimensionality of the dynamic programming algorithm.

Remark 5: Contrary to the standard LQG solution, the distribution of the estimation error $\Delta_{k}$ is not Gaussian. The distribution of $\Delta_{k}$ will have a support, which is a subset of $\left\{\Delta_{k} \mid \delta_{k}=0\right\}$.

Remark 6: Theorem 1 also holds for non-Gaussian noise input. The only assumptions needed in the proofs are that the noise sequence $\left(w_{k}\right)_{k}$ is i.i.d. and zero-mean and that its probability distribution exhibits certain symmetry and monotonicity properties.

\section{EXTENSION TO LOSSY NETWORKS}

The assumption of perfect transmission is difficult to sustain for many digital communication networks, in particular for wireless time-varying channels and random access schemes. In the following, we relax this strict requirement on the communication network by allowing packet dropouts to occur during transmission. In order to maintain the separation principle, we assume a TCP-like communication protocol as introduced in [18] for networked control systems. With respect to optimal control and scheduling schemes in lossy networks, the main feature of TCP-like communication protocols in the feedback link is that an acknowledgment is sent back to the sender, whenever a packet has been successfully transmitted. We consider the following network model: packet dropouts are modeled as a Bernoulli sequence $\left(q_{k}\right)_{k} \in\{0,1\}$ with a successful transmission probability $\mathrm{P}\left[q_{k}=1\right]=\alpha$ for any $k$ and

$$
q_{k}= \begin{cases}1 & \text { measurement successfully transmitted } \\ 0 & \text { packet dropout occurred }\end{cases}
$$

The system dynamics are equal to (1), but the measurement equation is now defined as

$$
y_{k}=q_{k} \delta_{k} x_{k} .
$$

The probability space $(\Omega, \mathcal{A}, \mathrm{P})$ is properly extended to incorporate the random sequence $\left(q_{k}\right)_{k}$. We assume that the acknowledgment is sent reliably and instantaneously to the scheduler. The scheduler knows at any time instance whether the measurement has been transmitted successfully. Therefore, the fact that the information patterns of controller and scheduler are nested, still holds.

It can be showed that basically all results in section III with perfect signal transmission carry over to the case of the proposed communication model with packet dropouts. The controller structure remains the same as in Equation (7). The estimator is given by

$\mathrm{E}\left[x_{k} \mid \mathcal{I}_{k}^{\mathcal{C}}\right]= \begin{cases}x_{k} & \text { for } \delta_{k}=1 \text { and } q_{k}=1 \\ \left(A-B L_{k}\right) \mathrm{E}\left[x_{k-1} \mid \mathcal{I}_{k-1}^{\mathcal{C}}\right] & \text { otherwise }\end{cases}$ 
The scheduler law is the solution of the following minimization problem with parameters given by Theorem 1:

$$
\begin{array}{r}
\min _{f} \mathrm{E}\left[\sum_{k=0}^{T-1}\left(1-q_{k} \delta_{k}\right) e^{\mathrm{T}} \Gamma_{k} e_{k}+\lambda \delta_{k}\right], \\
\text { s.t. } e_{k+1}=\left(1-q_{k} \delta_{k}\right) A e_{k}+w_{k} .
\end{array}
$$

Alike in Theorem 1, problem (16) can be solved by the dynamic programming algorithm, since $\left(q_{k}\right)_{k}$ is assumed to be i.i.d. .

In presence of time-delay during transmission, the property that the information patterns of scheduler and controller are nested does not hold and the separation property may not be established anymore. In this context, the notion of delayed information patterns introduced in [23] and the results therein might be useful to overcome this problem.

\section{THREE CLASSES OF SCHEDULERS}

The first class of schedulers are policies which are restricted to be causal and to have deterministic laws. These schedulers are called state-dependent schedulers in the following. For this class the optimal controller and scheduling policy has been found in previous sections. In order to show the effectiveness of the solution, we consider two other classes of schedulers, a deterministic scheduler and a purely randomized scheduler.

The deterministic scheduler determines the scheduling sequence offline. To find the optimal deterministic scheduler and optimal controller, it can be seen that separation holds, since for any fixed scheduling sequence, the controller is given by solving the standard linear quadratic problem for time-varying systems [17]. As the class of deterministic schedulers is a subset of the state-dependent class, the optimal deterministic scheduler can not perform better than the optimal state-dependent scheduler. The optimal scheduling sequence is determined by solving the following problem

$$
\min _{\left(\delta_{k}\right)_{k}} \sum_{k=1}^{T-1}\left(1-\alpha \delta_{k}\right) \operatorname{tr}\left[\Phi_{k} \Gamma_{k}\right]+\lambda \delta_{k}
$$

with

$$
\Phi_{k+1}=\left(1-\alpha \delta_{k}\right) A \Phi_{k} A^{\mathrm{T}}+C_{w}, \quad \Phi_{0}=C_{x_{0}},
$$

which can be computed by the deterministic dynamic programming algorithm.

The class of purely randomized schedulers is characterized by the property that their decision whether to transmit a measurement is given by an i.i.d. random variable, which is independent of any other variable. The scheduler is determined by the average transmission rate $\bar{\delta}=\mathrm{P}\left[\delta_{k}=1\right]=\mathrm{E}\left[\delta_{k}\right]$. Due to the fact that the scheduling sequence is a Bernoulli sequence, the problem separates into solving a linear quadratic problem of a jump linear system, see [24], resulting in (7) and then performing a line-search between zero and one to find the optimal transmission probability $\bar{\delta}$. In fact, the optimal

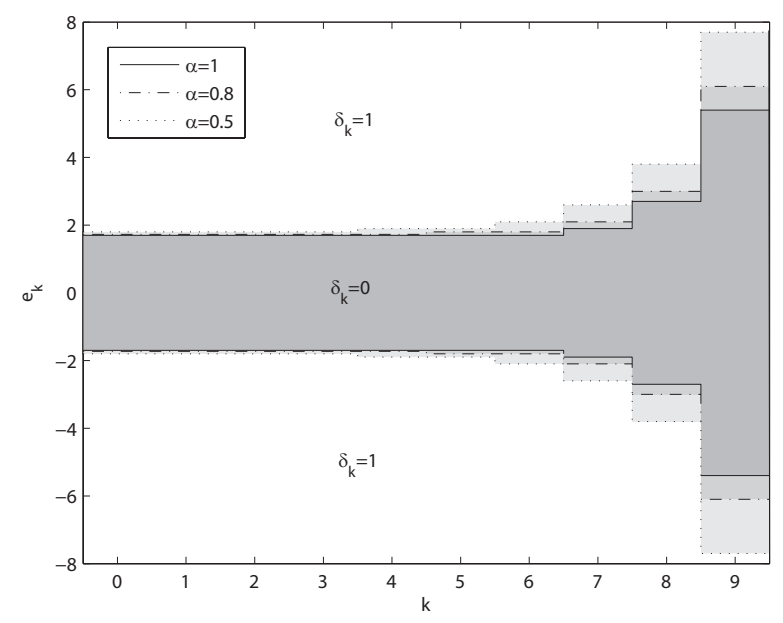

Fig. 2. Optimal state-dependent scheduling policy with indicated switching thresholds.

transmission rate is the solution of the following static optimization problem

$$
\min _{\bar{\delta} \in[0,1]}(1-\alpha \bar{\delta}) \sum_{k=1}^{T-1} \operatorname{tr}\left[\Phi_{k} \Gamma_{k}\right]+\lambda \bar{\delta}
$$

with

$$
\Phi_{k+1}=(1-\alpha \bar{\delta}) A \Phi_{k} A^{\mathrm{T}}+C_{w}, \quad \Phi_{0}=C_{x_{0}} .
$$

It can be easily shown that the cost function in (17) is convex with respect to the average transmission rate $\bar{\delta}$.

In all three classes, the problem of finding the optimal solution can be separated into an optimal linear quadratic regulator problem and an optimal estimation/scheduling problem. The optimal control law is equivalent for all three classes.

\section{NUMERICAL EXAMPLE}

We consider the scalar system as in (1) with $A=1$, $B=1, C_{w}=1$ and $C_{x_{0}}=1, \bar{x}_{0}=0$ with cost parameters $Q=Q_{T}=1, R=5$ and $T=10$. As already mentioned in section $\mathrm{V}$, the control law for the optimal solution is the same irrespective of the class of schedulers. Therefore, we can concentrate on the analysis of the estimation and communication cost $J^{S}$ given by Equation (8) with $\gamma^{*}$ defined in Lemma 2. The fixed cost $J^{\mathrm{C}}$ for above system parameters is 26.2 .

Figure 2 shows the optimal state-dependent scheduling policies given by the solution of optimization in (16) for successful transmission rate $\alpha=1$ (no packet dropouts), $\alpha=0.8$ and $\alpha=0.5$ with communication penalty $\lambda=$ 5. The lines indicate the threshold between $\delta_{k}=0$ and $\delta_{k}=1$ depending on $e_{k}$ which is given by Equation (16). Figure 2 shows that transmission occur sparsely, when packet dropouts are present. At first glance this seems to be counterintuitive, but can be reasoned by the fact that state updates at the controller are not guaranteed anymore, when $\delta_{k}=1$, in the presence of packet dropouts, whereas the price $\lambda$ has to be paid even when no updates reach the controller. 


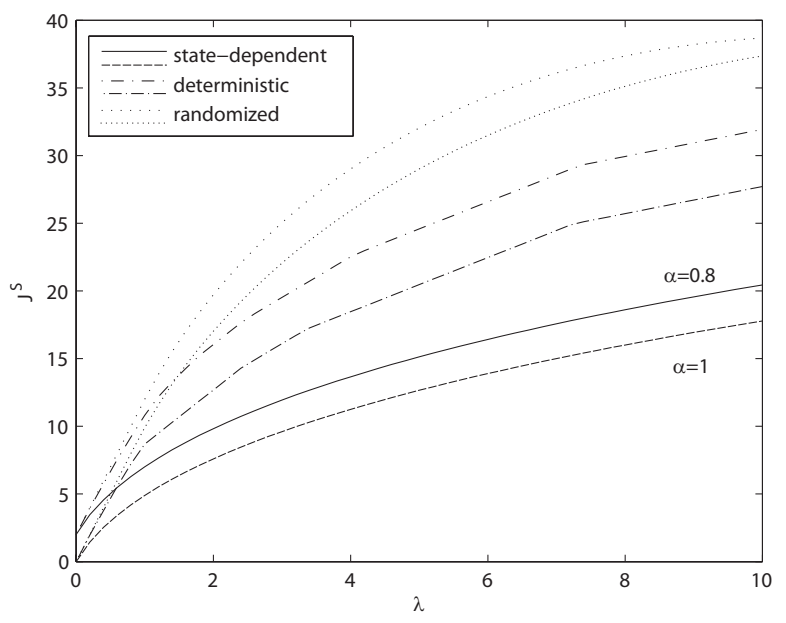

Fig. 3. Comparison of the three types of optimal schedulers with packet dropouts (upper curve) and without packet dropouts (lower curve) depending on communication penalty $\lambda$.

A comparison between the optimal schedulers of the three classes of schedulers introduced in section $\mathrm{V}$ is illustrated in Figure 3 without packet dropouts (lower curves) and with packet dropouts (upper curves) with dropout probability 0.2 $(\alpha=0.8)$. From Figure 3 we observe that packet dropouts in such range play a minor role in comparison to the choice of scheduling policy. The maximum decrease in cost is about $42 \%$, when comparing deterministic scheduler with the statedependent scheduler, and $55 \%$ for the randomized scheduler. In contrast to that the maximum performance gain of the state-dependent scheduler in a lossy network with $\alpha=0.8$ to a network fully reliable network $(\alpha=1)$ is about $15 \%$ for $\lambda$ greater than 1 . Hence, rather than increasing the reliability of the digital communication network, it can be conjectured that the choice of scheduling policy is a crucial factor for the performance under communication constraints.

\section{CONCLUSIONS}

By considering the LQG framework this paper solves the problem of joint optimal control and scheduling incorporating communication costs. It is showed that the problem can be separated into standard subproblems. These subproblems are computationally tractable in contrast to the initial joint optimization problem. An extension to TCPlike communication networks with packet dropouts is given and performance is compared with the optimal deterministic and optimal randomized scheduler with and without packet dropouts. Based on the theoretical findings in this paper, future work includes the extension to dynamical systems with noisy observations at the scheduler and the consideration of time delays during transmissions. In addition, ergodicity and stability issues still need to be discussed for the infinite horizon case. Finally, extensions of the proposed approach to networks with multiple sensor and controllers sharing a common network is planned.

\section{ACKNOWLEDGMENTS}

This work was supported in part by the German Research Foundation (DFG) within the Priority Program SPP 1305
"Control Theory of Digitally Networked Dynamical Systems". The authors would also like to thank the anonymous reviewers for their constructive comments, which helped to improve the quality of this paper.

\section{REFERENCES}

[1] H. Kushner, "On the optimum timing of observations for linear control systems with unknown initial state," Automatic Control, IEEE Transactions on, vol. 9, pp. 144-150, Apr 1964.

[2] M. Athans, "On the determination of optimal costly measurement strategies for linear stochastic systems," Automatica, vol. 8, no. 4, pp. 397-412, 1972.

[3] P. Seiler, Coordinated control of unmanned aerial vehicles. $\mathrm{PhD}$ thesis, Univ. California, Berkeley, 2001.

[4] R. Daoud, H. Amer, H. Elsayed, and Y. Sallez, "Ethernet-based car control network," in IEEE CCECE/CCGEI, (Ottawa), 2006.

[5] S. Hirche, M. Ferre, J. Barrio, C. Melchiorri, and M. Buss, "Bilateral control architectures for telerobotics," in Advances in Telerobotics, STAR series, pp. 163-176, Springer, 2007.

[6] S. Tatikonda, A. Sahai, and S. Mitter, "Stochastic linear control over a communication channel," Automatic Control, IEEE Transactions on, vol. 49, pp. 1549-1561, Sept. 2004.

[7] H. Rehbinder and M. Sanfridson, "Scheduling of a limited communication channel for optimal control," Automatica, vol. 40, no. 3, pp. 491-500, 2004.

[8] O. C. Imer and T. Basar, "Optimal estimation with limited measurements," Decision and Control, 2005 44th IEEE Conference on, vol. 2, pp. 1029-1034, 2005.

[9] O. C. Imer and T. Basar, "To measure or to control: optimal control with scheduled measurements and controls," American Control Conference, 2006. Proceedings of the 2006, pp. 1003-1008, June 2006.

[10] P. Bommannavar and T. Başar, "Optimal control with limited control actions and lossy transmissions," Decision and Control, 2008 47th IEEE Conference on, 09-11 Dec. 2008.

[11] M. Rabi, G. Moustakides, and J. Baras, "Multiple sampling for estimation on a finite horizon," Decision and Control, 2006 45th IEEE Conference on, pp. 1351-1357, Dec. 2006.

[12] M. Rabi, K. H. Johansson, and M. Johansson, "Optimal stopping for event-triggered sensing and actuation," Decision and Control, 2008 47th IEEE Conference on, 09-11 Dec. 2008.

[13] O. Imer and T. C. Basar, "Optimal control with limited controls," American Control Conference, 2006. Proceedings of the 2006, pp. 298-303, June 2006.

[14] Y. Xu and J. Hespanha, "Optimal communication logics in networked control systems," Decision and Control, 2004. CDC. 43rd IEEE Conference on, vol. 4, pp. 3527-3532 Vol.4, Dec. 2004.

[15] Y. Xu, Optimal Communication Logics in Networked Control Systems. $\mathrm{PhD}$ thesis, University of California, Santa Barbara, CA, USA, 2006.

[16] R. Cogill, S. Lall, and J. P. Hespanha, "A constant factor approximation algorithm for event-based sampling," Proceedings of the American Control Conference, no. 1, pp. 305-311, 2007.

[17] W. Wu and A. Arapostathis, "Optimal control of stochastic systems with costly observations - the general markovian model and the LQG problem," American Control Conference, 2005. Proceedings of the 2005, pp. 294-299 vol. 1, June 2005.

[18] B. Sinopoli, L. Schenato, M. Franceschetti, K. Poolla, and S. Sastry, "An LQG optimal linear controller for control systems with packet losses," Decision and Control, 2005 and 2005 European Control Conference. CDC-ECC '05. 44th IEEE Conference on, pp. 458-463, Dec. 2005.

[19] H. S. Witsenhausen, "A counterexample in stochastic optimum control," SIAM Journal on Control, vol. 6, no. 1, pp. 131-147, 1968.

[20] D. P. Bertsekas, Dynamic programming and optimal control. Vol. I. 3rd ed., Athena Scientific, Belmont, MA, 2005.

[21] Y. Bar-Shalom and E. Tse, "Dual effect, certainty equivalence, and separation in stochastic control," Automatic Control, IEEE Transactions on, vol. 19, pp. 494-500, Oct 1974.

[22] K. J. Åström, Introduction to Stochastic Control Theory. Dover Publications, 2006.

[23] B. Kurtaran, "Decentralized stochastic control with delayed sharing information pattern," Automatic Control, IEEE Transactions on, vol. 21, pp. 576-581, Aug 1976.

[24] O. Costa, M. Fragoso, and R. Marques, Discrete-Time Markov Jump Linear Systems. Springer London, 1st ed., 2005. 